\title{
A!
}

This is an electronic reprint of the original article.

This reprint may differ from the original in pagination and typographic detail.

Becerra, V. Fernández; Trif, Mircea; Hyart, Timo

\section{Topological charge, spin, and heat transistor}

Published in:

Physical Review B

DOI:

10.1103/PhysRevB.103.205410

Published: 10/05/2021

Document Version

Publisher's PDF, also known as Version of record

Please cite the original version:

Becerra, V. F., Trif, M., \& Hyart, T. (2021). Topological charge, spin, and heat transistor. Physical Review B, 103(20), [205410]. https://doi.org/10.1103/PhysRevB.103.205410

This material is protected by copyright and other intellectual property rights, and duplication or sale of all or part of any of the repository collections is not permitted, except that material may be duplicated by you for your research use or educational purposes in electronic or print form. You must obtain permission for any other use. Electronic or print copies may not be offered, whether for sale or otherwise to anyone who is not an authorised user. 


\title{
Topological charge, spin, and heat transistor
}

\author{
V. Fernández Becerra $\odot,{ }^{1, *}$ Mircea Trif, ${ }^{1}$ and Timo Hyart ${ }^{1,2}$ \\ ${ }^{1}$ International Research Centre MagTop, Institute of Physics, Polish Academy of Sciences, Aleja Lotnikow 32/46, PL-02668 Warsaw, Poland \\ ${ }^{2}$ Department of Applied Physics, Aalto University, 00076 Aalto, Espoo, Finland
}

(Received 9 December 2020; accepted 28 April 2021; published 10 May 2021)

\begin{abstract}
Spin pumping consists in the injection of spin currents into a nonmagnetic material due to the precession of an adjacent ferromagnet. In addition to the pumping of spin the precession always leads to pumping of heat, but in the presence of spin-orbital entanglement it also leads to a charge current. We investigate the pumping of charge, spin, and heat in a device where a superconductor and a quantum spin Hall insulator are in proximity contact with a ferromagnetic insulator. We show that the device supports two robust operation regimes arising from topological effects. In one regime, the pumped charge, spin, and heat are quantized and related to each other due to a topological winding number of the reflection coefficient in the scattering matrix formalism, translating to a Chern number in the case of Hamiltonian formalism. In the second regime, a Majorana zero mode switches off the pumping of currents owing to the topologically protected perfect Andreev reflection. We show that the interplay of these two topological effects can be utilized so that the device operates as a robust charge, spin, and heat transistor.
\end{abstract}

DOI: 10.1103/PhysRevB.103.205410

\section{INTRODUCTION}

Transistors are a celebrated example of a basic research discovery resulting in an enormous societal impact. They are the building blocks of the modern digital technology revolution owing to their ability to manipulate electrical currents with exponential dependencies on the control parameters [1]. Motivated by this success story, enormous amounts of research efforts have been devoted to enhancing the functionalities of the next generation of nanoelectronic devices by exploiting the various ways to manipulate the electrical currents on the level of single electrons [2-4], the spin degree of freedom [5-19], the thermal properties of mesoscopic structures [20-23], as well as the mutual coupling of charge, spin, and energy modes [24,25].

Topological materials are the gold standard for future electronic, spintronic, and heattronic devices, as the corresponding transport modes are intrinsically linked to each other in these systems [26,27]. This is best exemplified in the case of two-dimensional (2D) quantum spin Hall insulators (QSHI), which support one-dimensional helical edge modes, so that the electrons moving right and left carry opposite spins $[28,29]$. The QSHI states have been observed in various materials [30-32], but their potential for device applications is still waiting to be realized. Nevertheless, from the previously explored topological phenomena, quantum Hall effect, and ac Josephson effect, we know that topological effects are well suited for metrology applications. The quantum Hall has been widely used as a resistance standard [33] and ac Josephson effect as a voltage standard [34].

\footnotetext{
*becerra@magtop.ifpan.edu.pl
}

In this article, we consider a system where the QSHI edge is placed in proximity to a ferromagnetic insulator (FI) and a superconductor (SC) to realize Majorana zero-energy mode (MF) [35-37] (see Fig. 1). The technology for building this setup has already been developed, motivated by the prospects of utilizing MFs as a staple ingredient for topological quantum computers [38-42]. We explore the potential of this setup in a completely different context utilizing the perfect topologically protected Andreev reflection (AR) enabled by the MF $[37,43]$ but not exploiting the existence of a nonlocal quantum degrees of freedom used in topological quantum computers [44]. Namely, we study the charge, spin, and heat pumping in this system in the presence of precessing magnetization (see Fig. 1). The spin pumping is a scrutinized method to generate spin currents in magnetic heterostructures $[6-8,17]$ and forms the basis for many contemporary spintronic applications [45], but in the case of QSHIs there exists a unique property that both the charge and spin pumping are quantized and related to each other $[11,12]$. We show that also the heat current is quantized and elaborate the origin of these quantizations by showing that they result from a topological winding number of the reflection coefficient in the scattering matrix formalism and Chern number in the Hamiltonian formalism - resembling the quantized topological pumps proposed in other contexts [46-49]. Then we show that the key advantage of our QSHI-FI-SC heterostructure in comparison to the earlier proposals is that due to the presence of MF there exists also another topological operation regime, where the MF switches off the pumping of currents owing to the perfect AR. We show that it is possible to use external control parameters to tune between these operation regimes so that the device operates as a robust charge, spin, and heat transistor with exponential sensitivity on the applied gate voltage and precession angle. Moreover, two perfectly quantized limits 


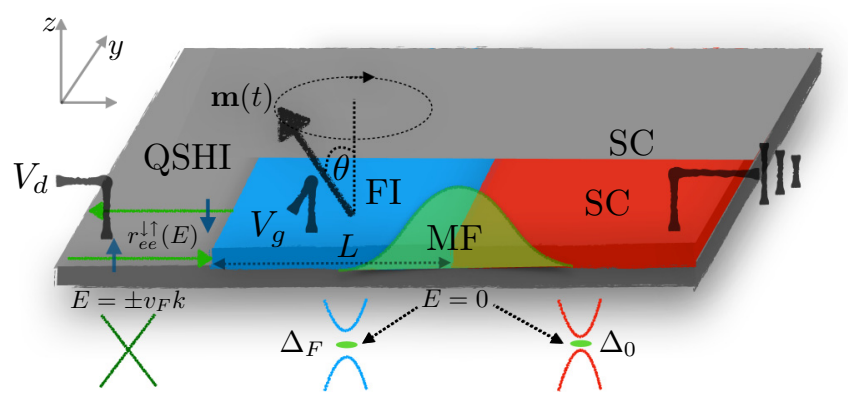

FIG. 1. Sketch of the proposed device consisting of a QSHI in proximity contact with FI of length $L$ (induced energy gap $\Delta_{F}=$ $|\mathbf{m}| \sin \theta$ ) and SC (induced energy gap $\Delta_{0}$ ). The monodomain magnetization $\boldsymbol{m}(t)$ precesses at an angle $\theta$ around the axis perpendicular to the QSHI driving charge, spin, and heat currents to the drain (left), which can be controlled with potential at the FI region $V_{g}$, the precession angle $\theta$, temperature and drain voltage $V_{d}$. The system harbors a zero-energy MF at the FI-SC interface that affects the pumped currents via the scattering coefficient $r_{e e}^{\downarrow \uparrow}(E)$.

allow us to build standards for the spin and heat pumping with the help of accurate measurement of the pumped electric charge.

\section{THEORETICAL APPROACH}

To describe the system, consisting of the edge states of 2D QSHI in proximity contact with FI and SC as illustrated in Fig. 1, we consider a time-dependent Bogoliubov-de Gennes (BdG) Hamiltonian,

$$
\mathcal{H}_{\mathrm{BdG}}(t)=\left[v_{F} p \sigma_{z}-\mu(x)\right] \tau_{z}+\boldsymbol{m}(x, t) \cdot \boldsymbol{\sigma}+\Delta(x) \tau_{x},
$$

where $\sigma=\left(\sigma_{x}, \sigma_{y}, \sigma_{z}\right)$ and $\boldsymbol{\tau}=\left(\tau_{x}, \tau_{y}, \tau_{z}\right)$ are Pauli matrices that act in the spin and Nambu space, respectively, $v_{F}$ is the Fermi velocity, $p=-i \hbar \partial_{x}$ is the momentum operator along the edge ( $x$ direction), $\boldsymbol{m}(x, t)$ is the time-dependent magnetization in the FI (which includes the exchange coupling strength between the two materials), $\Delta(x)$ is the induced superconducting order parameter, and $\mu(x)$ is the chemical potential. We assume that $\Delta(x)=\Delta_{0}$ is constant over the region occupied by the SC. The magnetization of the FI island is parametrized as $\boldsymbol{m}(x, t)=m_{0}(x)[\sin \theta(t) \cos \phi(t), \sin \theta(t) \sin \phi(t), \cos \theta(t)]$, where $m_{0}(x)=m_{0}$ under the FI region (uniform precession). Moreover, we consider periodic driving such that $\boldsymbol{m}(x, t+\mathcal{T})=\boldsymbol{m}(x, t)$, with $\mathcal{T}=2 \pi / \omega$ being the precession period and $\omega$ the precession frequency. Throughout the text we assume that the temperature is much smaller than the critical temperatures of the superconductivity and magnetism, so that the temperature dependence of $\Delta_{0}$ and $m_{0}$ can be neglected.

The dynamics of the magnetization in the FI results in pumping of charge, spin, and heat into the left lead. The pumped charge over one cycle in the adiabatic limit can be calculated from the expression [50,51]

$$
Q_{e}=-\frac{e}{4 \pi} \int d E\left(\frac{\partial f}{\partial E}\right) \int_{0}^{\mathcal{T}} d t \operatorname{Im}\left\{\operatorname{Tr}\left[\mathcal{S}^{\dagger} \tau_{z} \frac{\partial \mathcal{S}}{\partial t}\right]\right\},
$$

where $f(E)$ is the Fermi distribution function, and $\mathcal{S}(E, t) \equiv$ $\mathcal{S}(E, \theta(t), \phi(t))$ is the instantaneous scattering matrix pertaining to a normal metal-FI-SC junction. This can be casted in the form

$$
\mathcal{S}(E, \theta, \phi) \equiv\left(\begin{array}{ll}
\mathcal{S}^{e e}(E, \theta, \phi) & \mathcal{S}^{e h}(E, \theta, \phi) \\
\mathcal{S}^{h e}(E, \theta, \phi) & \mathcal{S}^{h h}(E, \theta, \phi)
\end{array}\right),
$$

accounting for both the normal (ee) and Andreev (eh) processes, so that each of these components is a matrix describing the spin-dependent scattering. The pumping of spin $S$ can be found analogously by using substitutions $e \rightarrow \hbar / 2$ and $\tau_{z} \rightarrow \sigma$ in Eq. (2). We consider only the $S_{z}$ component, since it is the only spin component conserved in the left lead. Finally, the heat $Q_{E}$ injected in the left lead is obtained from the expression

$$
Q_{E}=-\frac{\hbar}{8 \pi} \int d E\left(\frac{\partial f}{\partial E}\right) \int_{0}^{\mathcal{T}} d t \operatorname{Tr}\left[\frac{\partial \mathcal{S}}{\partial t} \frac{\partial \mathcal{S}^{\dagger}}{\partial t}\right]
$$

Here we have neglected the possible heat losses to the substrate. The spin-momentum locking in the QSHI edges limits the scattering matrix elements so that the only nonzero reflection coefficients are $r_{e e(h h)}^{\downarrow \uparrow}(E, \theta, \phi)$ and $r_{h e(e h)}^{\downarrow \uparrow}(E, \theta)$. Here, $r_{e e(h h)}^{\downarrow \uparrow}(E, \theta, \phi)$ describes the reflection amplitude for an electron (hole) with spin $\uparrow$ injected from the QSHI at energy $E$ to be reflected back to QSHI as electron (hole) with spin $\downarrow$ because of the FI and the SC. Similarly, $r_{h e(e h)}^{\downarrow \uparrow}(E, \theta)$ describe the amplitudes for the AR processes, where the electron (hole) is reflected back as a hole (electron). Each reflection coefficient accounts for all the possible scattering paths, including the effect of the MF at the FI-SC interface, and the reflection coefficients satisfy $\left|r_{e e(h h)}^{\downarrow \uparrow}(E, \theta, \phi)\right|^{2}+\left|r_{h e(e h)}^{\downarrow \uparrow}(E, \theta)\right|^{2}=1$.

The only $\phi$-dependent coefficients satisfy [52]

$$
r_{e e}^{\downarrow \uparrow}(E, \theta, \phi)=r_{0}(E, \theta) e^{i \phi}, r_{h h}^{\downarrow \uparrow}(E)=-\left[r_{e e}^{\downarrow \uparrow}(-E)\right]^{*},
$$

and the magnitude of $r_{e e}^{\downarrow \uparrow}(E, \theta, \phi)$ is suppressed at low energies due to the topologically protected perfect $\mathrm{AR} \mid r_{h e}^{\downarrow \uparrow}(E=$ $0, \theta) \mid=1$, so that for $E \ll m_{0}, \Delta_{0}$ it can be approximated as [52]

$$
\left|r_{0}(E, \theta)\right|^{2} \approx \frac{E^{2} / \Gamma^{2}}{1+E^{2} / \Gamma^{2}}
$$

where

$$
\Gamma=2 \Delta_{0}\left(\frac{\xi_{F}(0, \theta)}{\xi_{F}\left(V_{g}, \theta\right)}\right)^{2} \frac{\xi_{S}}{\xi_{F}\left(V_{g}, \theta\right)+\xi_{S}} e^{-2 L / \xi_{F}\left(V_{g}, \theta\right)}
$$

is the Majorana linewidth (for which $\left|r_{e e}^{\downarrow \uparrow}(E=\Gamma)\right|^{2}=$ $\left.\left|r_{h e}^{\downarrow \uparrow}(E)\right|^{2}=1 / 2\right)$ expressed in terms of the coherence lengths $\xi_{F}\left(V_{g}, \theta\right)=\hbar v_{F} / \sqrt{m_{0}^{2} \sin ^{2} \theta-\left(e V_{g}\right)^{2}}$ and $\xi_{S}=\hbar v_{F} / \Delta_{0}$ pertaining to the ferromagnet and superconductor, respectively. Here we have denoted the chemical potential in the FI region as $\mu_{F I}=e V_{g}$ to indicate that it can be controlled with the gate voltage and $L$ is the length of the FI region (Fig. 1). In analytic calculations we use the approximation (6), but numerical calculations are done using the full expression for $r_{e e}^{\downarrow \uparrow}(E, \theta, \phi)$ [52]. In the following we utilize the topological protection of the MFs, which ensures that $\Gamma$ depends exponentially on the parameters $V_{g}$ and $\theta$ [Eq. (7)]. This dependence only breaks down if $e V_{g} \rightarrow m_{0} \sin \theta$, at which point the electrons under 
the FI become gapless and the adiabatic scattering matrix approximation is no longer valid.

For simplicity, in the following we assume $\phi(t)=\omega t$ and $\theta(t) \equiv \theta$ is constant. However, many of our results can be generalized for arbitrary trajectory in $(\theta(t), \phi(t))$ space [52]. Interestingly, the special form of the scattering matrix for the combined system leads to the charge, spin, and heat pumped over a cycle to be determined by a single dimensionless charge $\mathcal{Q}$ :

$$
Q_{e}=e \mathcal{Q}, \quad S_{z}=-\frac{\hbar}{2} \mathcal{Q}, \quad Q_{E}=\frac{\hbar \omega}{2} \mathcal{Q} .
$$

In the adiabatic limit,

$$
\mathcal{Q}=-\frac{1}{2 \pi} \int d E\left(\frac{\partial f(E)}{\partial E}\right) \int_{0}^{2 \pi} d \phi\left|r_{e e}^{\downarrow \uparrow}(E, \theta, \phi)\right|^{2}
$$

depends on the applied drain voltage $V_{d}$ and temperature $k_{B} T$ $\left(k_{B}\right.$ is the Boltzmann constant) via $f(E)$, and the gate voltage $V_{g}$ and rotation angle $\theta$ via $\left|r_{e e}^{\downarrow \uparrow}(E, \theta, \phi)\right|^{2}$. In the special case of constant $\theta$ we can also go the rotating frame and calculate the frequency dependence of $\mathcal{Q}$ [52]. In the limits $T=0$ and $V_{d}=0$, we obtain

$$
\mathcal{Q}=\left[1-\frac{2 \Gamma}{\hbar \omega} \arctan \left(\frac{\hbar \omega}{2 \Gamma}\right)\right]
$$

In a continuous operation of the device the pumped charge, spin, and heat currents flowing into the drain can be written, respectively, as $\left\langle I_{e}\right\rangle=\omega Q_{e} / 2 \pi,\left\langle I_{s}\right\rangle=\omega S_{z} / 2 \pi$, and $\left\langle I_{E}\right\rangle=$ $\omega Q_{E} / 2 \pi$. If drain voltage is applied $\left(V_{d} \neq 0\right)$ there exist also dc current contributions which do not depend on the magnetization dynamics. In this case the pumped currents can be obtained by measuring the currents in the presence and absence of the magnetization dynamics [52].

\section{TOPOLOGICAL EFFECTS}

As shown above, all quantities of interest are determined by $\mathcal{Q}$. Before analyzing $\mathcal{Q}$ in detail, we first highlight the robust topological features of this quantity. Namely, we find that $\mathcal{Q}=0$ for $e V_{d}, k_{B} T, \hbar \omega \ll \Gamma$ owing to the perfect topological AR caused by the MF. On the other hand, $\mathcal{Q}=1$ if $e V_{d} \gg \Gamma$, or $k_{B} T \gg \Gamma$ or $\hbar \omega \gg \Gamma$. The latter quantization appears due to the topological winding of the phase of $r_{e e}^{\downarrow \uparrow}(E, \theta, \phi)=$ $r_{0}(E, \theta) e^{i \phi}$ when the above conditions guarantee that the magnitude satisfies $\left|r_{e e}^{\downarrow \uparrow}(E, \theta, \phi)\right|=1$. The quantization of the pumped charge to $\mathcal{Q}=1$ can also be understood as Thouless pumping arising due to a Chern number associated with the pumping cycle in the case of the Hamiltonian formalism [52]. The quantized charge and spin pumping in these limits are robust topological results which are independent of the details of the magnetization trajectory $(\theta(t), \phi(t))$ [52]. The topological protection guarantees that deviations of $\mathcal{Q}$ on the quantized values depend on an exponential way on the parameters $V_{g}$ and $\theta$.

On the other hand, the quantization of the heat $Q_{E}$ can be related to the mesoscopic charge relaxation in quantum capacitors [53,54]. There, the charge relaxation resistance associated to a single conduction channel coupled to the mesoscopic capacitor is $R_{q}=h / 2 e^{2}$ leading to $\left\langle I_{E}\right\rangle=R_{q}\left\langle I_{e}^{2}\right\rangle$. For circular precession, we find $\left\langle I_{e}^{2}\right\rangle=\left(\left\langle I_{e}\right\rangle\right)^{2}$, and hence the

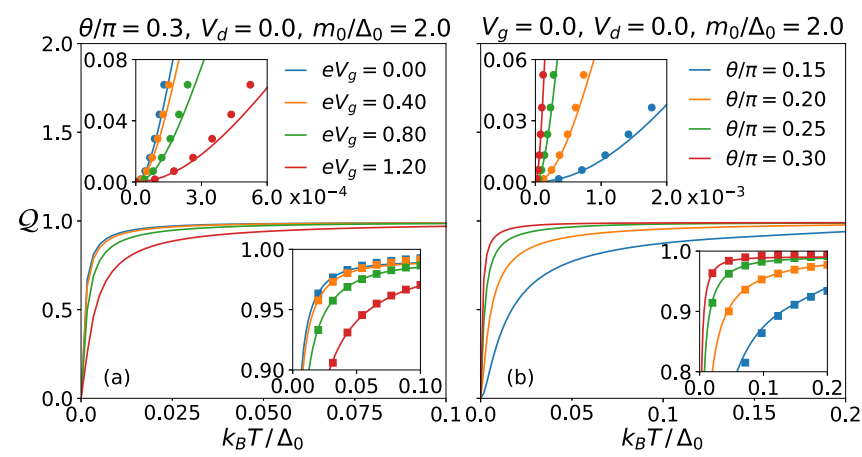

FIG. 2. The dimensionless charge $\mathcal{Q}$ in the adiabatic limit [Eq. (9)] as a function of $T$ for various values of (a) gate voltages $e V_{g}$ (in units $\Delta_{0}$ ) and (b) angles $\theta$. The insets are zooms into the suppression $\left(k_{B} T \ll \Gamma, \mathcal{Q} \approx 0\right)$ and quantization $\left(k_{B} T \gg \Gamma, \mathcal{Q} \approx 1\right)$ regimes. The asymptotic expressions of $\mathcal{Q}$ [Eq. (11)] are shown in the suppression (circles) and quantization (squares) regimes. The other parameters are $L=400 \mathrm{~nm}, \Delta_{0}=1 \mathrm{meV}, m_{0} / \Delta_{0}=2$, and $v_{F}=2.7 \times 10^{5} \mathrm{~m} / \mathrm{s}$.

quantization of the charge $Q_{e}$ engenders quantization of $Q_{E}$. Nevertheless, contrary to the mesoscopic capacitors, here the quantization stems from the topological gap of the system and not from the discreteness of the energy levels.

\section{TRANSISTOR BEHAVIOR}

The detailed analysis of the transistor characteristics can be based on the interplay of the topological effects discussed above. We start by discussing the characteristics in the absence of drain voltage $V_{d}=0$, because in this case the currents are caused purely by the magnetization dynamics.

In Fig. 2 we show the dimensionless charge $\mathcal{Q}$ in the adiabatic limit [Eq. (9)] as a function of temperature $T$ for different values of $V_{g}$ and $\theta$. The asymptotic expressions of $\mathcal{Q}$ at low and high temperatures are [52]

$$
\mathcal{Q} \approx\left\{\begin{array}{cc}
\frac{\pi^{2}}{3}\left(\frac{k_{B} T}{\Gamma}\right)^{2}, & k_{B} T \ll \Gamma \\
1-\frac{\pi}{4}\left(\frac{\Gamma}{k_{B} T}\right), & k_{B} T \gg \Gamma
\end{array} .\right.
$$

Due to the topological protection of MFs the Majorana linewidth $\Gamma$ depends exponentially on the control parameters $V_{g}$ and $\theta$ [Eq. (7)]. Therefore it is easy to see from Eq. (11) and Fig. 2 that the pumping can be efficiently turned on and of,f ensuring the device can operate as a transistor. Moreover, two perfectly quantized and extremely robust topological limits allow definition of standards for charge, spin, and heat pumping.

The operation of the transistor can also be controlled with the frequency. In Fig. 3 we show $\mathcal{Q}$ as a function of $\omega$. Again, we have two operation regimes, and the corresponding asymptotic expressions of $\mathcal{Q}$ are

$$
\mathcal{Q}=\left\{\begin{array}{ll}
\frac{1}{12}\left(\frac{\hbar \omega}{\Gamma}\right)^{2}, & |\hbar \omega| \ll \Gamma \\
1-\frac{\pi \Gamma}{\hbar|\omega|}, & |\hbar \omega| \gg \Gamma
\end{array},\right.
$$




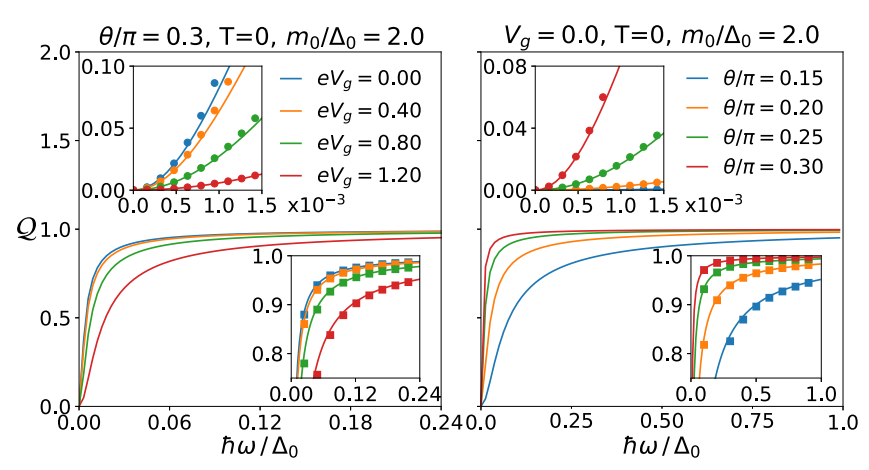

FIG. 3. The dimensionless charge $\mathcal{Q}$ as a function of $\omega$ for $V_{d}=$ $T=0$ and various values of (a) $e V_{g}$ (in units of $\Delta_{0}$ ) and (b) $\theta$. The insets are zooms into the suppression $(\hbar \omega \ll \Gamma, \mathcal{Q} \approx 0)$ and quantization $(\hbar \omega \gg \Gamma, \mathcal{Q} \approx 1)$ regimes. The asymptotic expressions of $\mathcal{Q}$ [Eq. (12)] are shown in the suppression (circles) and quantization (squares) regimes. The other parameters are the same as in Fig. 2.

demonstrating that pumping can again be turned on and off with the control parameters $V_{g}$ and $\theta$.

Finally, in Fig. 4 we show the dimensionless charge $\mathcal{Q}$ in the adiabatic limit [Eq. (9)] as a function of $V_{d}$. Similar robust switching behavior from $\mathcal{Q}=0$ to $\mathcal{Q}=1$ is obtained again with the asymptotic expressions,

$$
\mathcal{Q} \approx\left\{\begin{array}{ll}
\left(\frac{e V_{d}}{\Gamma}\right)^{2}, & e V_{d} \ll \Gamma \\
1-\left(\frac{\Gamma}{e V_{d}}\right)^{2}, & e V_{d} \gg \Gamma
\end{array},\right.
$$

indicating that $\mathcal{Q}$ depends exponentially on $V_{g}$ and $\theta$. We emphasize that for $V_{d} \neq 0$ also dc currents are present, and the expressions (13) and Fig. 4 only describe the contribution coming from the magnetization dynamics.

In addition to the low-energy continuum model, we have checked all features of the transistor behavior using the KWANT software package [55] implementation of the 2D quantum transport setup shown in Fig. 1, including realistic disorder potential. The results from the two methods show excellent agreement [52], solidifying the universality and robustness of the topological transistor. While the results have been obtained assuming circular precession of the FI magnetization around the $z$ axis, the quantization of the charge $Q_{e}$ and spin $S_{z}$ remain the same in the two topological limits, as long as the magnetization vector encloses the $z$ axis during the precession. On the other hand, the heat $Q_{E}$ will deviate from the universal expression in Eq. (8), since in this case $\left\langle I_{e}^{2}\right\rangle \neq\left(\left\langle I_{e}\right\rangle\right)^{2}[52]$.

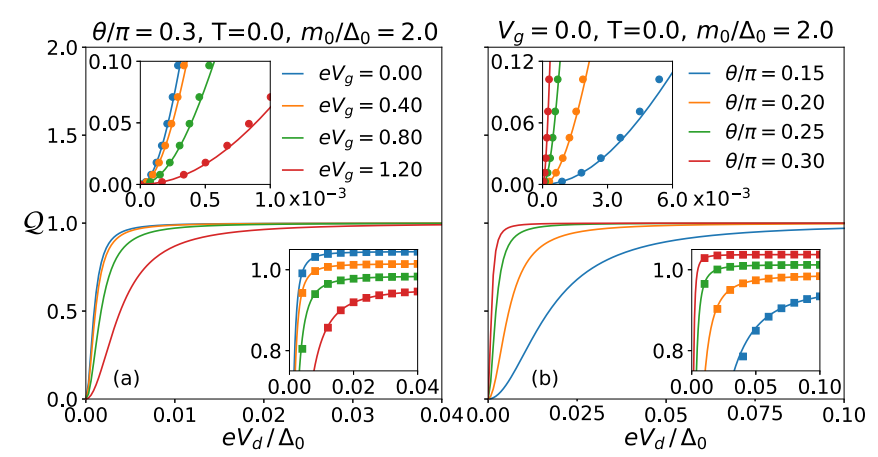

FIG. 4. The dimensionless charge $\mathcal{Q}$ as a function of drain voltage $V_{d}$ for various values of (a) gate voltages $V_{g}$ and (b) angles $\theta$. Two different limits for $\mathcal{Q}$ are evident in the plots. The insets are zooms into the suppression (low $V_{d}$ ) and quantization (high $V_{d}$ ) regimes, respectively. The asymptotic limits of the pumped charge are also shown in the suppression (circles) and quantization (squares) regimes. The charges in the bottom insets have been shifted evenly for the sake of visualization.

\section{CONCLUSIONS AND OUTLOOK}

We have described the operation principles of a robust charge, spin, and heat transistor consisting of a QSHI proximity coupled to FI and SC. The device supports two robust operation regimes arising from topological effects. In the suppression regime at low energies the pumping is switched off due to the perfect AR of the electrons impinging on the MF hosted in the device. Since the perfect AR is topologically protected, this suppression is not affected by disorder and other imperfections of the device. At high energies the pumped charge is quantized due to the topological winding number associated with the scattering matrix (or Thouless pumping). Therefore the operation in this regime is also intrinsically robust against imperfections. The operation frequencies in our analysis are limited by the energy gap of the system: $\hbar \omega \ll \Delta_{0}, m_{0} \sin \theta$. Thus the device can be operated at gigahertz frequencies, which is the typical frequency range of the spin-pumping experiments. Our device is scalable, as it is possible to pattern 2D QSHI with FI and SC arrays by various depositions methods.

\section{ACKNOWLEDGMENTS}

The work is supported by the Foundation for Polish Science through the IRA Programme cofinanced by the EU within the SG OP. Numerical simulations were carried out with the support of the Interdisciplinary Centre for Mathematical and Computational Modelling (ICM), University of Warsaw, under Grant No. G78-13.
[1] A. S. Sedra and K. C. Smith, Microelectronic Circuits, 5th ed. (Oxford University Press, Oxford, England, 2004).

[2] M. A. Kastner, The single-electron transistor, Rev. Mod. Phys. 64, 849 (1992).

[3] R. Hützen, A. Zazunov, B. Braunecker, A. L. Yeyati, and R. Egger, Majorana Single-Charge Transistor, Phys. Rev. Lett. 109, 166403 (2012).
[4] J. P. Pekola, O.-P. Saira, V. F. Maisi, A. Kemppinen, M. Möttönen, Y. A. Pashkin, and D. V. Averin, Single-electron current sources: Toward a refined definition of the ampere, Rev. Mod. Phys. 85, 1421 (2013).

[5] S. Datta and B. Das, Electronic analog of the electro-optic modulator, Appl. Phys. Lett. 56, 665 (1990). 
[6] A. Brataas, Y. Tserkovnyak, G. E. W. Bauer, and B. I. Halperin, Spin battery operated by ferromagnetic resonance, Phys. Rev. B 66, 060404(R) (2002).

[7] Y. Tserkovnyak, A. Brataas, and G. E. W. Bauer, Enhanced Gilbert Damping in Thin Ferromagnetic Films, Phys. Rev. Lett. 88, 117601 (2002).

[8] Y. Tserkovnyak, A. Brataas, G. E. W. Bauer, and B. I. Halperin, Nonlocal magnetization dynamics in ferromagnetic heterostructures, Rev. Mod. Phys. 77, 1375 (2005).

[9] A. Fert, Nobel Lecture: Origin, development, and future of spintronics, Rev. Mod. Phys. 80, 1517 (2008).

[10] P. A. Grünberg, Nobel Lecture: From spin waves to giant magnetoresistance and beyond, Rev. Mod. Phys. 80, 1531 (2008).

[11] X.-L. Qi, T. L. Hughes, and S.-C. Zhang, Fractional charge and quantized current in the quantum spin Hall state, Nat. Phys. 4, 273 (2008).

[12] F. Mahfouzi, B. K. Nikolić, S.-H. Chen, and C.-R. Chang, Microwave-driven ferromagnet-topological-insulator heterostructures: The prospect for giant spin battery effect and quantized charge pump devices, Phys. Rev. B 82, 195440 (2010)

[13] J. Wunderlich, B.-G. Park, A. C. Irvine, L. P. Zârbo, E. Rozkotová, P. Nemec, V. Novák, J. Sinova, and T. Jungwirth, Spin Hall effect transistor, Science 330, 1801 (2010).

[14] J. Liu, T. H. Hsieh, P. Wei, W. Duan, J. Moodera, and L. Fu, Spin-filtered edge states with an electrically tunable gap in a two-dimensional topological crystalline insulator, Nat. Mater. 13, 178 (2014).

[15] Y. Shiomi, K. Nomura, Y. Kajiwara, K. Eto, M. Novak, K. Segawa, Y. Ando, and E. Saitoh, Spin-Electricity Conversion Induced by Spin Injection into Topological Insulators, Phys. Rev. Lett. 113, 196601 (2014).

[16] A. A. Baker, A. I. Figueroa, L. J. Collins-McIntyre, G. van der Laan, and T. Hesjedal, Spin pumping in ferromagnettopological insulator-ferromagnet heterostructures, Sci. Rep. 5, 7907 (2015).

[17] J. Sinova, S. O. Valenzuela, J. Wunderlich, C. H. Back, and T. Jungwirth, Spin Hall effects, Rev. Mod. Phys. 87, 1213 (2015).

[18] J. Tian, S. Hong, I. Miotkowski, S. Datta, and Y. P. Chen, Observation of current-induced, long-lived persistent spin polarization in a topological insulator: A rechargeable spin battery, Sci. Adv. 3, e1602531 (2017).

[19] A. Hirohata, K. Yamada, Y. Nakatani, I.-L. Prejbeanu, B. Diény, P. Pirro, and B. Hillebrands, Review on spintronics: Principles and device applications, J. Magn. Magn. Mater. 509, 166711 (2020).

[20] F. Giazotto, T. T. Heikkilä, A. Luukanen, A. M. Savin, and J. P. Pekola, Opportunities for mesoscopics in thermometry and refrigeration: Physics and applications, Rev. Mod. Phys. 78, 217 (2006).

[21] J. P. Pekola, Towards quantum thermodynamics in electronic circuits, Nat. Phys. 11, 118 (2015).

[22] A. Fornieri and F. Giazotto, Towards phase-coherent caloritronics in superconducting circuits, Nat. Nanotechnol. 12, 944 (2017).

[23] D. Meidan, T. Gur, and A. Romito, Heat pumping from braiding Majorana zero modes, Phys. Rev. B 99, 205101 (2019).

[24] F. S. Bergeret, M. Silaev, P. Virtanen, and T. T. Heikkilä, Colloquium: Nonequilibrium effects in superconductors with a spin-splitting field, Rev. Mod. Phys. 90, 041001 (2018).
[25] R. Ojajärvi, J. Manninen, T. T. Heikkilä, and P. Virtanen, Nonlinear spin torque, pumping, and cooling in superconductor/ferromagnet systems, Phys. Rev. B 101, 115406 (2020).

[26] M. Z. Hasan and C. L. Kane, Colloquium: Topological insulators, Rev. Mod. Phys. 82, 3045 (2010).

[27] X.-L. Qi and S.-C. Zhang, Topological insulators and superconductors, Rev. Mod. Phys. 83, 1057 (2011).

[28] C. L. Kane and E. J. Mele, Quantum Spin Hall Effect in Graphene, Phys. Rev. Lett. 95, 226801 (2005).

[29] B. A. Bernevig, T. L. Hughes, and S.-C. Zhang, Quantum spin Hall effect and topological phase transition in $\mathrm{HgTe}$ quantum wells, Science 314, 1757 (2006).

[30] M. König, S. Wiedmann, C. Brüne, A. Roth, H. Buhmann, L. W. Molenkamp, X.-L. Qi, and S.-C. Zhang, Quantum spin Hall insulator state in HgTe quantum wells, Science 318, 766 (2007).

[31] L. Du, I. Knez, G. Sullivan, and R.-R. Du, Robust Helical Edge Transport in Gated InAs/GaSb Bilayers, Phys. Rev. Lett. 114, 096802 (2015).

[32] S. Wu, V. Fatemi, Q. D. Gibson, K. Watanabe, T. Taniguchi, R. J. Cava, and P. Jarillo-Herrero, Observation of the quantum spin Hall effect up to 100 kelvin in a monolayer crystal, Science 359, 76 (2018).

[33] J. Weis and K. von Klitzing, Metrology and microscopic picture of the integer quantum Hall effect, Philos. Trans. R. Soc. A 369, 3954 (2011).

[34] C. A. Hamilton, Josephson voltage standards, Rev. Sci. Instrum. 71, 3611 (2000).

[35] L. Fu and C. L. Kane, Superconducting Proximity Effect and Majorana Fermions at the Surface of a Topological Insulator, Phys. Rev. Lett. 100, 096407 (2008).

[36] L. Fu and C. L. Kane, Josephson current and noise at a superconductor/quantum-spin-Hall-insulator/superconductor junction, Phys. Rev. B 79, 161408(R) (2009).

[37] C. Beenakker, Search for Majorana fermions in superconductors, Annu. Rev. Condens. Matter Phys. 4, 113 (2013).

[38] S. Hart, H. Ren, T. Wagner, P. Leubner, M. Mühlbauer, C. Brüne, H. Buhmann, L. W. Molenkamp, and A. Yacoby, Induced superconductivity in the quantum spin Hall edge, Nat. Phys. 10, 638 (2014).

[39] V. S. Pribiag, A. J. A. Beukman, F. Qu, M. C. Cassidy, C. Charpentier, W. Wegscheider, and L. P. Kouwenhoven, Edge-mode superconductivity in a two-dimensional topological insulator, Nat. Nanotechnol. 10, 593 (2015).

[40] Y. Liu, S. Vaitiekènas, S. Martí-Sánchez, C. Koch, S. Hart, Z. Cui, T. Kanne, S. A. Khan, R. Tanta, S. Upadhyay, M. E. Cachaza, C. M. Marcus, J. Arbiol, K. A. Moler, and P. Krogstrup, Semiconductor-ferromagnetic insulatorsuperconductor nanowires: Stray field and exchange field, Nano Lett. 20, 456 (2020).

[41] S. Vaitiekenas, Y. Liu, P. Krogstrup, and C. M. Marcus, Zerofield topological superconductivity in ferromagnetic hybrid nanowires, Nat. Phys. 17, 43 (2021).

[42] R. M. Lutchyn, E. P. A. M. Bakkers, L. P. Kouwenhoven, P. Krogstrup, C. M. Marcus, and Y. Oreg, Majorana zero modes in superconductor-semiconductor heterostructures, Nat. Rev. Mater. 3, 52 (2018).

[43] K. T. Law, P. A. Lee, and T. K. Ng, Majorana Fermion Induced Resonant Andreev Reflection, Phys. Rev. Lett. 103, 237001 (2009). 
[44] C. Nayak, S. H. Simon, A. Stern, M. Freedman, and S. Das Sarma, Non-Abelian anyons and topological quantum computation, Rev. Mod. Phys. 80, 1083 (2008).

[45] A. Hoffmann and S. D. Bader, Opportunities at the frontiers of spintronics, Phys. Rev. Appl. 4, 047001 (2015).

[46] D. J. Thouless, Quantization of particle transport, Phys. Rev. B 27, 6083 (1983).

[47] J. E. Avron, A. Raveh, and B. Zur, Adiabatic quantum transport in multiply connected systems, Rev. Mod. Phys. 60, 873 (1988).

[48] J. E. Avron and M. C. Cross, Integer charge transport in Josephson junctions, Phys. Rev. B 39, 756 (1989).

[49] R.-P. Riwar, M. Houzet, J. S. Meyer, and Y. V. Nazarov, Multi-terminal Josephson junctions as topological matter, Nat. Commun. 7, 11167 (2016).

[50] M. V. Moskalets, Scattering Matrix Approach to Non-Stationary Quantum Transport (World Scientific, London, 2012).
[51] M. Blaauboer, Charge pumping in mesoscopic systems coupled to a superconducting lead, Phys. Rev. B 65, 235318 (2002).

[52] See Supplemental Material at http://link.aps.org/supplemental/ 10.1103/PhysRevB.103.205410 for more details regarding: 1) the Thouless-like treatment of pumping (appendices A, G and $\mathrm{H}), 2$ ) the full scattering problem and the asymptotic limits (appendices B and C), 3) the 2D lattice simulations (appendices D and E), and 4) the rotating wave description (appendix F).

[53] M. Büttiker, H. Thomas, and A. Prêtre, Mesoscopic capacitors, Phys. Lett. A 180, 364 (1993).

[54] S. E. Nigg, R. López, and M. Büttiker, Mesoscopic Charge Relaxation, Phys. Rev. Lett. 97, 206804 (2006).

[55] C. W. Groth, M. Wimmer, A. R. Akhmerov, and X. Waintal, KWANT: A software package for quantum transport, New J. Phys. 16, 063065 (2014). 